\title{
TO STUDY THE EFFECT OF BETA BLOCKER (CARVEDILOL) IN THE MANAGEMENT OF HEART FAILURE
}

\author{
Dr Namala Balakrishna*1 ${ }^{1}$, Dr. Souris Kondaveti ${ }^{2}$, Dr. Ramamohan Pathapati ${ }^{3}$ \\ ${ }^{1}$ Junior Resident, Dept. of Pharmacology, Osmania Medical College, Koti, Hyderabad, Telangana, India, \\ ${ }^{2}$ Assistant Professor, Dept. of Pharmacology, Osmania Medical College, Koti, Hyderabad, Telangana, India \\ ${ }^{3}$ Associate Professor, Dept. of pharmacology, Narayana Medical College, Nellore, Andhra Pradesh. India
}

\begin{abstract}
:
Background and objectives: Congestive heart failure (CHF) continues to be a major clinical and public health problem with shift in etiology from hypertension, valvular heart disease to ischaemia. Carvedilol is a non-selective beta-blocker with $\alpha 1$ blocking activity. In the present study, the efficacy of Carvedilol, by assessing the baseline left ventricular ejection fraction heart rate, and blood pressure.

Materials and methods: A total number of 40 patients were enrolled in the study, 20 were heart failure patients and 20 were age-sex matched controls (not receiving carvedilol). After the study period of 24 weeks, the efficacy of carvedilol in reducing heart rate, systolic blood pressure, diastolic blood pressure and left ventricular ejection fraction were evaluated.

Results: Carvedilol when given in low doses, was effective in increasing left ventricular ejection fraction from $(31.73 \pm 2.28$ $\%)$ to $(40.85 \pm 7.51 \%)$ with $\mathrm{p}<0.0001$, decreasing heart rate from $(80.95 \pm 5.10)$ to $(71.50 \pm 6.11)$ beats $/ \mathrm{min}$, with $\mathrm{p}<0.0001$, and reducing systolic blood pressure $5.25 \pm 4.99$ with $\mathrm{p}<0.0006$ and diastolic blood pressure $6.50 \pm 6.71$ with $\mathrm{p}<0.4077$

Conclusion: Carvedilol is a non-selective beta-blocker with $\alpha 1$ - blocking activity and when given in low doses, increases cardiac ejection fraction, decreases ventricular mass, improves the shape of the ventricle, and reduces systolic and diastolic volumes. In conclusion from our study, Carvedilol increases the baseline left ventricular ejection fraction and heart rate more significantly in the patients than in the controls.

Keywords: Congestive heart failure (CHF), Carvediloleffect on blood pressure, increases cardiac ejection fraction
\end{abstract}

\section{INTRODUCTION}

Congestive heart failure (CHF) continues to be a major clinical and public health problem with shift in etiology from hypertension, valvular heart disease to ischemia. It is a condition that can result from any structural or functional cardiac disorder that impairs the ability of the heart to fill with or pump a sufficient amount of blood through the body. The most common cause is coronary heart disease ${ }^{1}$ (CHD), especially myocardial infarction (MI).Others include hypertension, valve disease, cardiomyopathy and arrhythmias, particularly atrial fibrillation $^{2,3}$ Infections, anaemia and thyrotoxicosis can exacerbate, but also occasionally cause heart failure. Independent risk factors include diabetes, smoking, obesity, left ventricular hypertrophy or asymptomatic left ventricular dysfunction ${ }^{2,4}$. Heart failure is most commonly due to an enlarged and poorly contracting left ventricle, known as systolic heart failure 5, 6 Reduced left ventricular emptying leads to a fall in cardiac output, which triggers compensatory mechanisms. These effects paradoxically have a role in the progression of chronic heart failure ${ }^{6}$ and contribute to common symptoms Such as dyspnoea oedema, fatigue, lethargy and exercise intolerance. Heart failuremay lead to various complications, including arrhythmias; thromboembolism and pulmonary congestion atrial fibrillation (AF) may be the cause or consequence of heart failure and is present in about a third of patients. Treatment of CHF aims to relieve symptoms, maintain a euvolemic state and to improve prognosis by delaying progression of heart failure and reducing cardiovascular risk. Drugs used include: diuretic agents, vasodilator agents, positive inotropes, ACE inhibitors, beta blockers, and aldosterone antagonists. Beta blockers have been evaluated in nearly 10, 000 patients with chronic heart failure in over 20 randomised clinical trials and have been shown to decrease the risk of death and the need for hospital admission and to improve patients clinical status. These benefits were attained in patients already taking ACE inhibitors and diuretics (with or without digitalis), with ischemic and non-ischemic disease and with different degrees of functional impairment. Until recently, beta blockers were contraindicated in the treatment of CHF, largely because heart failure was viewed primarily as a hemodynamic disorder.

*For Correspondence Dr Namala Balakrishna Dept. of Pharmacology, Osmania Medical College,

Koti, Hyderabad, Telangana, India, Email: balunamala@gmail.com, drsourisuresh@gmail.com Mobile: 09492577777 
Although drugs with positive inotropic effects can produce short-termsymptomatic improvements in patients with heart failure, their long-term use does not prolong life ${ }^{7}$.

As the failing heart depends on $\beta$-adrenergic support to maintain its performance, acute pharmacological effects of any antiadrenergic compound may induce myocardial depression and a decrease in cardiac output. Although such effects may be counteracted by the vasodilator properties of some $\beta$ - blocking drugs, which by reducing systemic vascular resistance could counteract myocardial depression, an abrupt suppression of adrenergic support may initially precipitate a clinical worsening of heart failure. Nevertheless, this negative pharmacological response is transitory and manageable by administration of initial low doses and gradual dosage increase ${ }^{8}$. Additionally, chronic effects of $\beta$-blocking agents differ from the acute effects because they result essentially from the inhibition of neurohormonal responses that aggravate heart failure, favourably changing myocardium biology ${ }^{9]}$.It has been recently hypothesized that heart failure is an entity with a progressive decline in ventricular function due to progressive myocyte dysfunction (caused by gene expression changes), loss of cells (due to necrosis and apoptosis), and subsequent cell and cardiac chamber remodelling ${ }^{[10]}$. The remodelling process results in ventricular enlargement, an increase in wall stress, relative myocardial ischemia, energy depletion, progressive interstitialfibrosis, and additional activation of sympathetic nervous and renin angiotensin-aldosterone systems ${ }^{10}$. Cardiac sympathetic activity increase and elevated neither plasma nor epinephrine levels are reactions that occur early in heart failure patients. They may be already detected in asymptomatic left ventricular dysfunction ${ }^{11}$ and increase as the syndrome progresses ${ }^{12}$. Sympathetic activation leads to an increase in heart rate, arteriolar vasoconstriction, and peripheral vascular resistance and to a decrease in blood flow and sodium excretion consequently increasing ventricular volume and pressure. Cardiac work and oxygen consumption increase. Nor epinephrine may induce myocardial hypertrophy, but reduces coronary circulation's ability to adequately supply blood to an enlarged ventricular wall, leading to myocardial ischemia ${ }^{13}$. Additionally, norepinephrine exerts direct toxic effects on the myocardium, causing myocyte dysfunction and necrosis by several mechanisms. $\beta 1$-and $\beta 2$-receptor stimulation provokes cyclic adenosine monophosphate (cAMP)mediated calcium overload in cardiac myocytes, and activates calcium-dependent ATPases, decreasing the availability of highlyenergetic phosphates, further worsening mitochondrial function ${ }^{14}$. In addition, by stimulating growth andoxidative stress in terminally differentiated cells, norepinephrine can trigger apoptosis ${ }^{15}$ Several studies have demonstrated that elevated cardiac sympathetic activity is one of the leading causes of the progressive decline in myocardial function and of a poor prognosis in heart failure patients ${ }^{16}$.Hence the recognition of deleterious effects provoked by the sympathetic nervous system in left ventricular systolic function and the potential inhibition of sympathetic-adrenergic stimulationby chronic therapy with $\beta$-blocking drugs led to an increased administration of such drugs in heart failure management.Carvedilol is a nonselective beta-adrenoreceptor antagonist and an alpha1-adrenoreceptor antagonist and a potent antioxidant. It has no intrinsic sympathomimetic activity. The beta-blocking actions of carvedilol are generally evident in humans within one hour of administration, and the alpha-mediated vasodilatory effects, manifested by decreased peripheral resistance and decreased blood pressure, are evident within about 30 minutes of administration. At high dosages, carvedilol exerts calcium channel blocking activity. It also has significant antioxidant properties. Carvedilol inhibits the generation of oxygen free radicals and prevents low-density lipoprotein (LDL) oxidation, which, in turn, reduces the uptake of LDL into the coronary vasculature. This antioxidant activity may contribute to carvedilol's cardio protective effects. Carvedilol improves left ventricular function (LVEF) and to reduce mortality. The starting dosage of carvedilol is $3.125 \mathrm{mg}$ orally twice daily for two weeks. Carvedilol should be taken with food to reduce the incidence of orthostatic hypotension. Patients should be observed for adverse reactions, especially dizziness, light headedness and hypotension, for one hour after the first dose and again after each dosage increase. Blood pressure should be measured with the patient standing. Patients should be instructed to weigh themselves every day and to contact their physician immediately if they experience a weight gain. The dosage should be doubled every two weeks to the maximum dosage or the highest tolerated dosage. The maximum recommended dosage for carvedilol is $25 \mathrm{mg}$ twice daily in patients weighing less than $85 \mathrm{~kg}$ and $50 \mathrm{mg}$ twice daily in patients weighing $85 \mathrm{~kg}$ or more.

\section{AIMS AND OBJECTIVES}

\section{$\checkmark \quad$ To study the effect of beta blocker on the left ventricular ejection fraction. \\ $\checkmark \quad$ To describe its effect on the heart rate. \\ $\checkmark$ To observe the effect of Carvedilol on blood pressure.}

\section{MATERIALS AND METHODS}

The study was conducted with the joint collaboration of M.G.M Hospital and the Department of Pharmacology, Kakatiya Medical College, Warangal. The study protocol was approved by the institutional ethical committee. All the subjects enrolled into the study gave written informed consent for their participation. All male and female patients between 30 to 85 years of age, diagnosed with heart failure falling into the category of functional New York Heart Association (NYHA) class II or III with left ventricular ejection fraction (LVEF) $\leq$ $35 \%$ were included in the study. Patients were excluded if they had a history of drug sensitivity or allergic reaction to alpha or beta-blockers, contraindication or intolerance to beta-blockers, pregnant or lactating women and women planning to become pregnant, history of asthma or severe chronic obstructive pulmonary disease; severe liver or kidney diseases, patients unwilling to provide written informed consent 
second-degree or third degree heart block and sick sinus syndrome.

A total number of 40 patients were enrolled in the study, 20 in control group (patients not receiving carvedilol or placebo, but were receiving therapy as prescribed by the physician) and 20 in carvedilol group. All the patients were evaluated for demographic data, laboratory profile, clinically features and non invasively for LV function by echo cardiogram (for ejection fraction) at baseline and at end of treatment after 24 weeks

\section{Statistical Analysis}

Data was entered into excel spread sheet-7. Continuous data was described as mean and standard deviation and unpaired and paired $t$ test was used to infer the data between the two groups and within groups respectively. A two tailed $p$ value $<0.05$ was considered statistically significant. Sample size was calculated based on an increase in LVEF by $3.5 \%$. with $80 \%$ power and onesided alpha error of 0.05 . A total of sixteen patients per group will be required. To account for lost to follow-up of patients we considered 20 patients per group.

\section{RESULTS}

In the Beta Blocker study group out of total of 40 subjects, 20 were heart failure patients and 20 were age and gender matched controls. There were 13 males and 7 females in the Carvedilol group and 14 males and 6 females in the control group. Evaluation of the base line demographic parameters of the Beta blocker wing shows that there is no statistically significant difference;

Age (56.80 \pm 3.41 vs. $60.10 \pm 6.3$ yrs; $p=0.0613)$;

Height $(1.59 \pm 0.06$ vs. $1.58 \pm 0.06 \mathrm{~m} ; \mathrm{p}=0.4613)$;

Weight $(65.55 \pm 4.45$ vs. $61.55 \pm 5.27 \mathrm{~kg}$; $\mathrm{p}=0.5376)$; and

Body mass index $\left(24.75 \pm 2.82\right.$ vs. $24.84 \pm 3.10 \mathrm{~m} / \mathrm{kg}^{2}$; $\mathrm{p}=0.9339$ );

Between the Carvedilol group and the control group respectively. (Table-1)

Table 1: Demographic data

\begin{tabular}{|l|l|l|l|}
\hline & Carvedilol & Control & p-Value \\
\hline Gender & M-13 / F-7 & M-14 / F-6 & 1.000 \\
\hline Age $(\mathrm{yrs})$ & $56.80 \pm 3.41$ & $60.10 \pm 6.3$ & 0.0613 \\
\hline Height $(\mathrm{m})$ & $1.59 \pm 0.06$ & $1.58 \pm 0.06$ & 0.4613 \\
\hline Weight $(\mathrm{kg})$ & $65.55 \pm 4.45$ & $61.55 \pm 5.27$ & 0.5376 \\
\hline Body Mass Index cm/ $/ \mathrm{kg}^{2}$ & $24.75 \pm 2.82$ & $24.84 \pm 3.10$ & 0.9339 \\
\hline
\end{tabular}

Table-2shows that the mean change in the heart rate in the Carvedilol group is significant with $(9.45 \pm 7.25)$ beats / $\mathrm{min}$; to a change of $(3.20 \pm 3.16)$ beats / $\mathrm{min}$ in the control group, with $\mathrm{p}=0.0024$ (Graph -1$)$.
Likewise the effect on the left ventricular ejection fraction is also more significant in the Carvedilol group than the control group, with a change from (-9.12 \pm $7.33)$ vs. $(-3.43 \pm 2.94)$ respectively (Graph-2).

Table 2: Carvedilol Vs Control

\begin{tabular}{|l|l|l|l|l|}
\hline S.NO & VARIABLE & Carvedilol Group & Control Group & p-Value \\
\hline 1. & Heart Rate & $9.45 \pm 7.25$ & $3.20 \pm 3.16$ & 0.0024 \\
\hline 2. & Systolic Blood Pressure & $5.25 \pm 4.99$ & $13.75 \pm 9.01$ & 0.0006 \\
\hline 3. & Diastolic Blood Pressure & $6.50 \pm 6.71$ & $8.50 \pm 7.45$ & 0.4077 \\
\hline 4. & Left Ventricular Ejection Fraction & $-9.12 \pm 7.33$ & $-3.43 \pm 2.94$ & 0.0017 \\
\hline
\end{tabular}

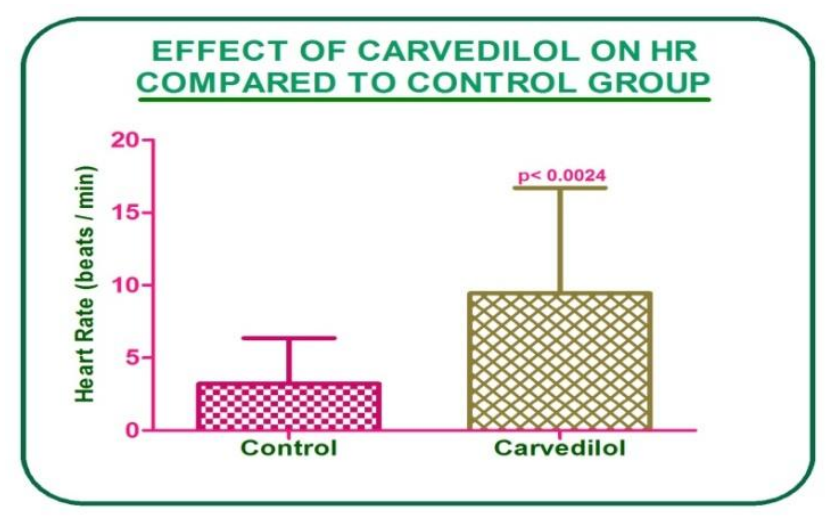

Graph 1
EFFECT OF CARVEDILOL ON LVEF COMPARED TO CONTROL GROUP

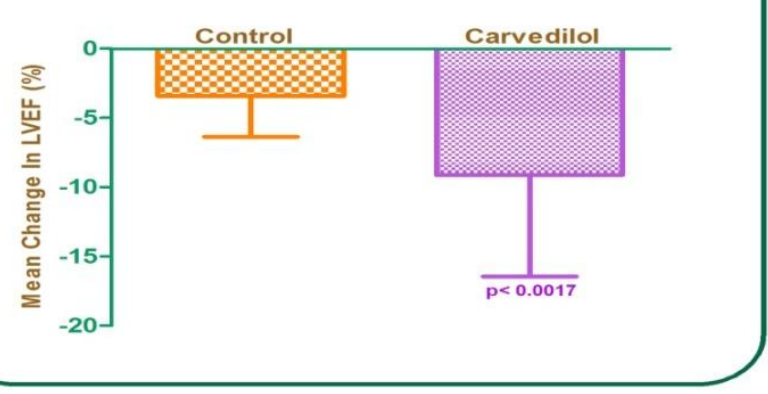

Graph 2 
Table-3 shows the per se effect of Beta blocker (Carvedilol) which is shown to decrease the heart rate from $(80.95 \pm 5.10)$ to $(71.50 \pm 6.11)$ beats / min, with $\mathrm{p}<0.0001$, compared to decrease from $(77.50 \pm 4.10)$ to $(74.30 \pm 3.15)$ beats / min, with $\mathrm{p}<0.0002$, in the control group (Graph-3).
It is also seen that it significantly raises the left ventricular ejection fraction from $(31.73 \pm 2.28 \%)$ to $(40.85 \pm 7.51 \%)$ with $\mathrm{p}<0.0001$, in comparison to an increase from $(30.35 \pm 3.7)$ to $(33.78 \pm 3.55)$ in the control group (Graph-4).

Table 3: Effect of Carvedilol

\begin{tabular}{|c|c|c|c|c|c|c|c|}
\hline \multirow{2}{*}{ S.N. } & \multirow{2}{*}{ VARIABLE } & \multicolumn{3}{|c|}{ Carvedilol Group } & \multicolumn{3}{c|}{ Control Group } \\
\cline { 3 - 8 } & & Before & After & p-Value & Before & After & p-Value \\
\hline 1. & Heart Rate & $80.95 \pm 5.10$ & $71.50 \pm 6.11$ & $<0.0001$ & $77.50 \pm 4.10$ & $74.30 \pm 3.15$ & 0.0002 \\
\hline 2. & Systolic Blood Pressure & $132.0 \pm 8.94$ & $126.75 \pm 10.04$ & 0.0002 & $133.0 \pm 9.23$ & $\begin{array}{c}119.25 \pm \\
10.55\end{array}$ & $<0.0001$ \\
\hline 3. & Diastolic Blood Pressure & $86.00 \pm 6.81$ & $79.50 \pm 6.05$ & 0.0004 & $81.50 \pm 7.45$ & $73.0 \pm 4.7$ & $<0.0001$ \\
\hline 4. & $\begin{array}{c}\text { Left Ventricular Ejection } \\
\text { Fraction }\end{array}$ & $31.73 \pm 2.28$ & $40.85 \pm 7.51$ & $<0.0001$ & $30.35 \pm 3.7$ & $33.78 \pm 3.55$ & $<0.0001$ \\
\hline
\end{tabular}

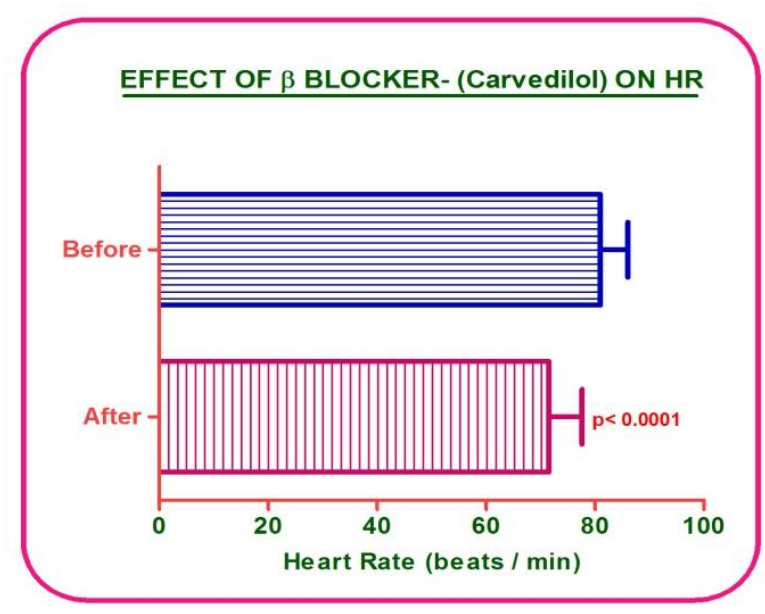

Graph 3

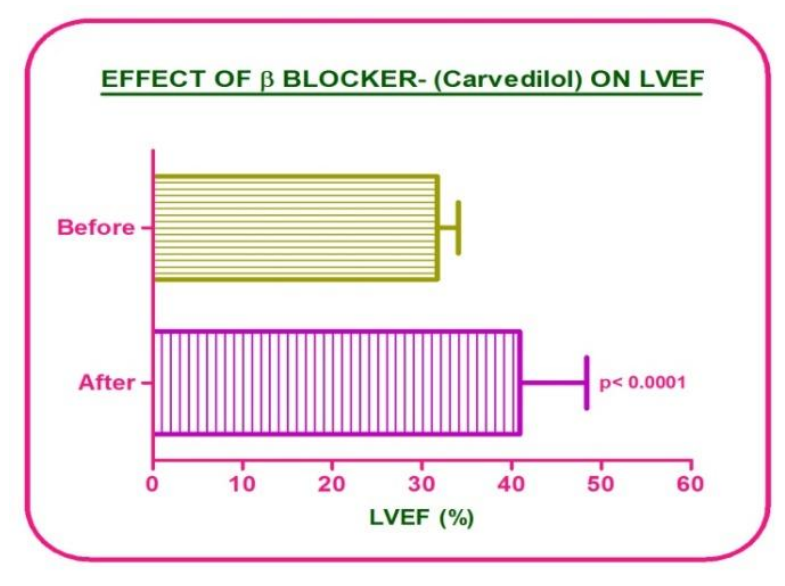

Graph 4

\section{DISCUSSION}

The present study was conducted with the evaluation of the effects of the beta blocker which was most commonly prescribed in our setup in heart failure condition, which in our case turned out to be Carvedilol.

For the Beta blocker wing, the patients who were prescribed Carvedilol were followed up for a period of six months after drug titration, and their effects on heart rate, systolic blood pressure, diastolic blood pressure and left ventricular ejection fraction were studied.
Carvedilol is a non-selective beta-blocker with $\alpha_{1}$ blocking activity and when given in low doses, this beta-blocker increases cardiac ejection fraction, decreases ventricular mass, improves the shape of the ventricle, and reduces systolic and diastolic volumes which was substantiated in our study with Carvedilol, wherein we found that the increase in the baseline left ventricular ejection fraction and heart rate was much more significant in the patients than in the controls.

Although the specific effects of the sympathetic nervous system on the progression of heart failure are unclear, beta-blockers appear to confer benefits by inhibiting a host of effects from sympathetic activation. For example, sympathetic activation can cause peripheral vasoconstriction, impair sodium excretion by the kidneys, provoke arrhythmias, stimulate growth and oxidative stress in terminally differentiated cells, and trigger programmed cell death. All of these harmful effects are mediated via the $\alpha_{1}, \beta_{1}$, and $\beta_{2}$-adrenergic receptors, at which beta-blockers exert their effects.

These effects of the beta blockers can be elucidated by the long term usage of the drug, with large sample size and long duration study design and the ensuing effects commented upon. But our study was too small both in terms of subjects enrolled and the duration, for commenting on the statistical significance of these effects. But most of the subjects who have been enrolled have reported reduced symptoms, improved clinical status and decreased incidence of hospitalization.

Our study helps to contribute to the data that is already available and also to compare it with the relevant guidelines to make our approach more current in the pharmacotherapeutic considerations of the management of patients with heart failure.

\section{CONCLUSION}

Treating heart failure is a challenge to the physician because of its rising incidence, high rate of hospitalization and mortality and frequent co-morbid disease and drug interactions. Carvedilol is a nonselective beta-blocker with $\alpha_{1}$ - blocking activity and when given in low doses, this beta-blocker increases ISSN: 2250-1177 
cardiac ejection fraction, and reduces systolic and diastolic volumes which were substantiated in our study.

One of the next steps towards improving heart failure management is to investigate the reasons underlying the low use of $b$ blockers in general practice and then to address these issues with educational programs. Such programs are clearly needed to improve heart failure management in general practice and should give special consideration to the role of b-blockers in the treatment

\section{REFERENCES}

1. Fox KF, Cowie MR, Wood DA, Coats AJ, Gibbs JS, Eur Heart J 2001; 22 : 228-36.

2. Lip GYH, Gibbs CR, et al. BMJ,2000; 320: 104-107.

3. McKelvie RS, Benedict CR, et al. BMJ,1999; 318: 14001402 .

4. Kenchaiah S, Narula J, Vasan RS, Med Clin North Am,2004; 88:1145-72.

5. Coats AJS.Medicine, 1998; 26: 116-121.

6. McDonagh TA, Dargie HJ. Medicine, 1998; 26: 111-115.

7. Watson RDS, Gibbs CR, et al. BMJ,2000; 320: 236-239.

8. Hall S, Cigarroa CG, Marxoux L, et al. J Am CollCardiol,1995; 25: 1154-61.

9. Bristow MR, Gilbert EM. Eur Heart J,1995; 16(suppl F): 20 31.

10. Eichorn EJ, Bristow MR. Circulation, 1996; 94: 2285-96.

11. Rendqvist B, Elam M, Bergmann-Sverrisdottir Y, Eisenhofer G, Friberg P.Circulation, 1997; 95: 169-75.

12. Bristow MR. N Engl J Med 1984; 311: 850-1.

13. Anversa P, Ricci R, Olivetti G. J Am CollCardiol,1986; 7 : 1140-9. of CHF. Effective implementation of these treatments on an individual basis should greatly improve the outcome of patients who develop heart failure.

\section{ACKNOWLEDGMENT}

I am much honored to thank my institution, M.G.M Hospital \& Kakatiya Medical College, for granting this work. I want to thank also the participants for their willingness to participate in the study by understanding the benefit from it.

14. Mann DL, Kent RL, Pardons B, Cooper IV. Circulation,1992; 85: 790- 804

15. Communal C, Singh K, Pimentel DR, Collucci WS. Circulation, 1998; 98: 1329-34.

16. Cohn JN, Levine TB, Olivari MT, et al. N Engl J Med,1984; 311: 819-23.

17. Braunwald"s Heart disease A text book of cardiovascular Medicine 8th page4, edition chapter 23 page 563

18. Braunwald $\mathrm{E}$ heart failure; an overview in fishma AP, editor heart failure,Newyork, McGraw-hill 1977.

19. Denolin $\mathrm{h}$ et al the definition of heart failure,Eur heart $\mathrm{J}$, 1983,4-445-46.

20. Ho KK, Pinsky JL, Kannel WB, Levy D. The epidemiology of heart failure:the Framingham Study. J Am CollCardiol, 1993;22(Suppl A):6A-13A.

21. GOODMAN AND GILMANS the pharmacological basis of therapeutics, 11 edition page 869-895.

22. American Heart Association Heart disease and strokestatistics 2004 update, DallasTexas American Heart Association 2003 pp 1-48.

23. www.acc.org heart failure guidelines. 